PROCEEDINGS OF THE

AMERICAN MATHEMATICAL SOCIETY

Volume 115, Number 3, July 1992

\title{
$K$-THEORETICAL INDEX THEOREMS FOR GOOD ORBIFOLDS
}

\author{
CARLA FARSI
}

(Communicated by Palle E. T. Jorgensen)

\begin{abstract}
In this note we study index theory for general and good orbifolds. We prove a $K$-theoretical index theorem for good orbifolds, and from this we deduce as a corollary a numerical index formula.
\end{abstract}

Let $D$ be a pseudodifferential elliptic operator on the closed orbifold $Q$. In $\S 1$ we give an index formula involving a certain class $[\widetilde{D}]$ associated to $D$. In $\$ 2$ we prove a $K$-theoretical index theorem (in analogy with the main theorem in [9]) for good orbifolds (a good orbifold is an orbifold that can be covered by a smooth manifold), which relates the class $[\widetilde{D}]$ with the class of its symbol. It is also natural to consider in this case, besides the usual (analytical) index of $D$, its Atiyah-Singer index $[2,12]$. We then recover from the $K$-theoretical index theorem the main theorem in [2]. In $\S 3$ we relate the analytical and the Atiyah-Singer indices of $D$.

\section{AN ORBIFOLD INDEX THEOREM}

We first give an index theorem for general orbifolds $Q$. We can suppose throughout this paper that $Q$ is even-dimensional, since the case in where the dimension of $Q$ is odd can be obtained from this by crossing with $\mathrm{T}$. All the orbifolds are also assumed to be orientable and closed. Note that any nonorientable orbifold is always finitely covered by an orientable orbifold.

Every orbifold $Q$ arises as a quotient $Q=P / G$, where $G$ is a compact group acting locally freely on the smooth manifold $P$ [11]. In our case we can choose $G=\operatorname{SO}(q)$, where $q$ is the dimension of $Q$ and $P$ is the orthonormal frame bundle of $Q$.

Let $\eta^{(0)}$ and $\eta^{(1)}$ be two orbifold vector bundles over $Q$. We say that $D$ is an elliptic pseudodifferential operator on $Q$ acting from the $L^{2}$-sections $\Gamma\left(\eta^{(0)}\right)$ of the bundle $\eta^{(0)}$ to the $L^{2}$-sections $\Gamma\left(\eta^{(1)}\right)$ of $\eta^{(1)}$ if on each orbifold chart $U_{i} \approx \mathbf{R}^{q} / G_{i}$ the lift of $D$ to $\mathbf{R}^{q}$ is a pseudodifferential elliptic operator. We assume throughout this paper that $D$ has order 0 . (We can always reduce to this case.) In analogy with the manifold case, every section of $\eta^{(0)}$ that is in $\operatorname{Ker}(D)$ is $C^{\infty}$ and so is every section of $\eta^{(1)}$ in $\operatorname{Ker}\left(D^{*}\right)$. This is because we only use local properties of $D$. Also $\operatorname{Ker}(D)$ and $\operatorname{Coker}(D)$ are finite-dimensional, so

Received by the editors December 5, 1990.

1991 Mathematics Subject Classification. Primary 46L80, 57S25.

Key words and phrases. $K$-theory, orbifolds, index, Gauss-Bonnet. 
that [12]

$$
\operatorname{Ind}_{a}(D) \underset{\text { def }}{=} \operatorname{Dim}(\operatorname{Ker}(D))-\operatorname{Dim}(\operatorname{Coker}(D)) .
$$

We define $C(P) \rtimes G$ to be the orbifold $C^{*}$-algebra $C^{*}(Q)$ [6]. The element $[\widetilde{D}] \in K K\left(C^{*}(Q), \mathbf{C}\right)$, defined in [6], coincides with the element associated to the lift $\widetilde{D}$ of $D$ to $P$. Therefore we can consider the image of $[\widetilde{D}]$ in the cyclic cohomology group $H C^{e v}\left(C^{\infty}(P \rtimes G)\right)$ via $c h^{*}$. As remarked by Connes at the end of $\S 8$ in [5], $C^{\infty}(G)$ embeds in $C^{\infty}(P \rtimes G): C^{\infty}(G) \stackrel{r}{\hookrightarrow} C^{\infty}(P \rtimes G)$ and the restriction $r^{*} c h^{*}([\widetilde{D}])$ of $c^{*}([\widetilde{D}])$ to $C^{\infty}(G)$ is given exactly by $r^{*} c h^{*}([\widetilde{D}])=$ $S^{l} \chi, q=2 l=\operatorname{dim} Q$, where $\chi$ is the distributional index character of $\widetilde{D}$ defined by Atiyah in [1], i.e., $\chi \in H C^{0}\left(C^{\infty}(G)\right)$,

$$
\chi(f) \underset{\text { def }}{=} \operatorname{Tr}\left(\begin{array}{c}
\text { action of } \\
f \text { in } \operatorname{ker} \widetilde{D}
\end{array}\right)-\operatorname{Tr}\left(\begin{array}{c}
\text { action of } \\
f \text { in } \operatorname{ker} \widetilde{D}^{*}
\end{array}\right)
$$

and $S$ is an operator defined by Connes in [5]. Therefore,

Theorem 1. Let $D$ be a pseudodifferential elliptic operator on the $\operatorname{spin}^{c}$ orbifold $Q$. Then

$$
\operatorname{Ind}_{a}(D)=\left\langle c h^{*}[\widetilde{D}], r_{*}(\mathbf{1})\right\rangle,
$$

where $r: C^{\infty}(G) \hookrightarrow C^{\infty}(P \rtimes G)$ is the canonical embedding, $\mathbf{1} \in H C_{e v}\left(C^{\infty}(G)\right)$ is the element corresponding to the constant function 1 , and $r_{*}: H C_{e v}\left(C^{\infty}(G)\right)$ $\rightarrow H C_{e v}\left(C^{\infty}(P \rtimes G)\right)$ is the induced homomorphism.

Proof. $\operatorname{Ind}_{a}(D)=\langle\chi, \mathbf{1}\rangle, \chi \in H C^{0}\left(C^{\infty}(G)\right), \mathbf{1} \in H C_{e v}\left(C^{\infty}(G)\right)$ by [11]. Note that 1 corresponds to the function $1 \in C^{\infty}(G)^{G} \equiv H C_{e v}\left(C^{\infty}(G)\right)$ by [4]. Because $\langle$,$\rangle is S$-invariant [5],

$$
\operatorname{Ind}_{a}(D)=\left\langle r^{*} c h^{*}[\tilde{D}], \mathbf{1}\right\rangle=\left\langle c h^{*}[\tilde{D}], r_{*}(\mathbf{1})\right\rangle \text {. }
$$

When $G$ acts freely on $P$ this is the Atiyah-Singer index theorem [3] (c.f. $[5, \S 6$, Theorem 5]).

\section{GOOD ORBIFOLDS}

In the case of a good orbifold (i.e., its universal cover is a smooth manifold) another definition of index for a pseudodifferential elliptic operator $D$ is possible (see $[2,12])$. In fact, let $\widehat{Q}$ be the universal cover of $Q, \pi_{1}^{\mathrm{ORB}}(Q)=\Gamma$ be the fundamental group of $Q$, and $\widehat{D}$ be the lift of $D$ to $\widehat{Q}$. Then $\widehat{Q} / \Gamma=Q$ and $\Gamma$ acts on $\widehat{Q}$ properly. The Atiyah-Singer index of $D$, AS-index, is defined as follows (c.f. [2]). On $\widehat{Q}$ we consider a $\Gamma$-invariant positive measure $d \hat{\mu}$ (the lift of a positive measure $d \mu$ on $Q$ ). Let $\hat{\eta}^{(i)}, i=0,1$, be the bundle over $\widehat{Q}$ lift of the bundle $\eta^{(i)}, i=0,1$ over $Q$. Note also that $L^{2}\left(\hat{\eta}^{(0)} \oplus \hat{\eta}^{(1)}\right) \cong L^{2}(\widehat{Q}) \times \operatorname{End}\left(\mathbf{C}^{n}\right), \quad n=\operatorname{Dim}\left(\hat{\eta}^{(0)}\right)+\operatorname{Dim}\left(\hat{\eta}^{(1)}\right)$, with the action of $\Gamma$ trivial on $\operatorname{End}\left(\mathbf{C}^{n}\right)$. The bounded operators on $L^{2}\left(\hat{\eta}^{(0)} \oplus \hat{\eta}^{(1)}\right)$ that commute with the action of $\Gamma$ form a von-Neumann algebra $A(\hat{\eta})$ that has a natural trace function denoted by $\operatorname{Tr}_{\Gamma}$. In particular if $P \in A(\hat{\eta})$ is an orthogonal projection onto a subspace $H$ of $L^{2}\left(\hat{\eta}^{(0)} \oplus \hat{\eta}^{(1)}\right)$, so that $H$ is a $\Gamma$ module, we define

$$
\operatorname{Dim}_{\Gamma}(H) \underset{\text { def }}{=} \operatorname{Tr}_{\Gamma}(P) \in \mathbf{R}
$$


Applying this to $\operatorname{Ker}(\widehat{D})$ and $\operatorname{Coker}(\widehat{D})$ we get a finite real-valued index

$$
\text { AS-ind }(D) \underset{\text { def }}{=} \operatorname{Dim}_{\Gamma}\left(\operatorname{Ker}(\widehat{D})-\operatorname{Dim}_{\Gamma}(\operatorname{Coker}(\widehat{D}))\right. \text {. }
$$

Next we will define the $K$-theoretical $\Gamma$-index of $D$. Firstly we will rewrite the orbifold, $C^{*}$-algebra $C^{*}(Q)$, up to Morita equivalence.

Proposition 2. Let $Q$ be a good orbifold. Then $C_{0}(\widehat{Q}) \rtimes \Gamma$ and $C^{*}(Q)$ are Morita equivalent.

Proof. Let $\widehat{P}$ be the orthogonal frame bundle of $\widehat{Q}$. The following diagram

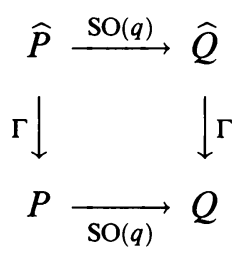

commutes. Since $\Gamma$ and $\mathrm{SO}(q)$ act freely on $\widehat{P}$ and their actions commute, by a theorem of P. Green (see [15]), $C^{*}(\widehat{P} / \Gamma, \mathrm{SO}(q))$ is Morita equivalent to $C^{*}(\widehat{P} / \mathrm{SO}(q), \Gamma)$. class

Hence an elliptic pseudodifferential elliptic operator $D$ on $Q$ determines a

$$
[\widehat{D}] \in K K\left(C_{0}(\widehat{Q}) \rtimes \Gamma, \mathbf{C}\right) \cong K K_{\Gamma}\left(C_{0}(\widehat{Q}), \mathbf{C}\right) .
$$

To define the $K$-theoretical $\Gamma$-index $\operatorname{IND}_{a}(\widehat{D})$ of $D$, which is an element of $K_{0}\left(C^{*}(\Gamma)\right)$, we first observe that since $\Gamma$ acts on $L^{2}\left(\hat{\eta}^{(i)}\right)$, so also $C^{*}(\Gamma)$ does, in a canonical way. Now $\widehat{D}$ is a Fredholm operator between $L^{2}\left(\eta^{(0)}\right)$ and $L^{2}\left(\eta^{(1)}\right)$, and so it determines an element of $K_{0}\left(C^{*}(\Gamma)\right)$, which we call $\operatorname{IND}_{a}(\widehat{D})$ (c.f. $\left.[10, \S 4]\right) . \operatorname{IND}_{a}(\widehat{D})$ is represented by the projections of $L^{2}\left(\eta^{(0)}\right)$ onto $\operatorname{Ker}(\widehat{D})$ and of $L^{2}\left(\eta^{(1)}\right)$ onto $\operatorname{Ker}\left(\widehat{D}^{*}\right)$ The following theorem can be recovered from a theorem of Kasparov [9].

Theorem 3. Let $D$ be a pseudodifferential elliptic operator on the good orbifold $Q, D: L^{2}\left(\eta^{(0)}\right) \rightarrow L^{2}\left(\eta^{(1)}\right)$. Let $\widehat{Q}$ be the universal cover of $Q, \Gamma=\pi_{1}^{\mathrm{ORB}}(Q)$, and $\widehat{D}$ be the operator $D$ lifted to $\widehat{Q}$. Then,

$$
\operatorname{IND}_{a}(\widehat{D})=\operatorname{IND}_{t}(\widehat{D}) \text { in } K K\left(\mathbf{C}, C^{*}(\Gamma)\right) \text {, }
$$

with

$$
\operatorname{IND}_{t}(\widehat{D}) \underset{\operatorname{def}}{=}[C] \bigotimes_{C_{0}(\widehat{Q}) \rtimes \Gamma} j_{\Gamma}([\widehat{D}]),
$$

where $[\widehat{D}] \in K K_{\Gamma}\left(C_{0}(\widehat{Q}), \mathbf{C}\right), j_{\Gamma}: K K_{\Gamma}(A, B) \rightarrow K K(A \rtimes \Gamma, B \rtimes \Gamma)$ is the canonical homomorphism, and $[C] \in K_{0}\left(C_{0}(\widehat{Q}) \rtimes \Gamma\right)$ is determined by the projection $p(x, g)=\sqrt{c(x) c\left(g^{-1} x\right)}$, where $c \in C_{c}^{\infty}(\widehat{Q})$ is such that $\int_{\Gamma} c(x g) d g=1$ and where $c \geq 0$.

Note that we could also have an index theorem with coefficients in a $C^{*}$ bundle rather than in a vector bundle in the spirit of [14].

As a corollary to Theorem 3 we obtain. 
Theorem 4. Let $D, Q, \widehat{Q}, \Gamma$, and $\widehat{D}$ be as in Theorem 3. Then,

$$
\tau\left(\operatorname{IND}_{a}(\widehat{D})\right)=\operatorname{AS} \text {-ind }(D),
$$

where $\tau$ is the canonical trace on $K_{0}\left(C^{*}(\Gamma)\right)=\left(\right.$ Idempotents of $\left.C^{*}(\Gamma) \otimes \mathscr{K}\right) / \sim$. Proof. $\tau$ is given by $\tau(a \otimes A)=\tau^{\Gamma}(a) \otimes T(A)$ where $a \in C^{*}(\Gamma), a=$ $\sum_{g \in \Gamma} \lambda_{g}[g], \lambda_{g} \in \mathbf{R}, \tau^{\Gamma}(a)=\lambda_{e}, e=$ unit of $\Gamma, A \in \mathscr{K}=\mathscr{K}\left(L^{2}(Q)\right), T=$ canonical trace on $\mathscr{K}\left(L^{2}(Q)\right) \subseteq \mathscr{B}\left(L^{2}(Q)\right)$. This trace coincides with the trace in $[2$, p. 57$]$.

\section{RELATIONS BETWEEN INDICES}

As we have seen in $\S 1$ and in $\S 2$, if $Q$ is a good orbifold and $D$ is a pseudodifferential elliptic operator on $Q$, then we can define the two indices $\operatorname{Ind}_{a}(D)$ and AS-ind $(D)$. The first one is necessarily an integer, while the second one is a rational number. In general they do not coincide, but there is an interesting relation between them, which is a corollary of the main theorems in [13] and [2] (c.f. also [12, III]). In fact Atiyah's argument applies also to the case in where the action is not free.

Theorem 5. Let $D, Q, \widehat{Q}, \Gamma$, and $\widehat{D}$ be as in Theorem 3. Then,

$$
\operatorname{Ind}_{a}(D)=\mathrm{AS}-\operatorname{ind}(D)+R \text {, }
$$

where (with the notation as in the introduction in [13]),

$$
R=\sum_{i=1}^{c} \int \frac{1}{m_{i}}\left((-1)^{\left|\Sigma_{i}\right|}\left\langle c h^{\Sigma}(D) \mathscr{T}^{\Sigma}(Q),\left[\Sigma_{i}\right]\right\rangle\right),
$$

with $\Sigma_{i}$ running over the strata of $Q$.

For example, if $\mathscr{E}$ is the Euler operator on $Q$, then $\operatorname{Ind}_{a}(\mathscr{E})$ is equal to the Euler characteristic of $Q$ as a vector space (c.f. [11, PROPOSITION]) and AS-ind $(\mathscr{E})=X_{S}(Q)$, where $X_{S}(Q)$ is the Euler-Satake characteristic of $Q$, by the Gauss-Bonnet theorem for orbifolds of Satake [16] and the general formula in [12]. Since $R$ depends only on the singular structure of $Q$, it follows that $R$ is 0 if $Q$ is a smooth manifold, and so in that case we recover the main theorem in [2] from Theorem $5, \operatorname{Ind}_{a}(D)=\mathrm{AS}$-ind $(D)$.

We would like to thank Professor J. Rosenberg for helpful conversations and the referee for useful comments and suggestions.

\section{REFERENCES}

1. M. F. Atiyah, Elliptic operators and compact groups, Lecture Notes in Math., vol. 401, Springer-Verlag, Heidelberg and New York, 1974.

2. __ Elliptic operators, discrete groups and von Neumann algebras, Asterisque 32-33 (1976), 43-72.

3. M. F. Atiyah and I. Singer, The index of elliptic operators III, Ann. of Math. (2) 87 (1968), 546-604.

4. J. L. Brylinski, Algebras associated with group actions and their homology, preprint.

5. A. Connes, The Chern character in K-homology, part I, Non commutative differential geometry, Inst. Hautes Études Sci. Publ. Math. 62 (1986), 257-360. 
6. C. Farsi, K-theoretical index theorems for orbifolds, Quart. J. Math. Oxford Ser. (2) (to appear).

7. M. Hilsum, Opérateurs de signature sur un variété lipschitzienne et modules de Kasparov non bornés, C. R. Acad. Sci. Paris Sér. I Math. 297 (1983), 49-52.

8. G. G. Kasparov, Topological invariants of elliptic operators I: K-homology, Math. USSR.Izv. 9 (1975), 751-792.

9. __ An index for invariant elliptic operators, $K$-theory, and representations of Lie groups, Soviet Math. Dokl. 27 (1983), 105-109.

10. __ Operator $K$-theory and its applications: elliptic operators, group representations, higher signatures, $C^{*}$-extensions, Proc. Internat. Congr. of Mathematicians, Aug. 16-24, 1983, Warsaw, 1983, pp. 987-1000.

11. T. Kawasaki, The signature theorem for $V$-manifolds, Topology 17 (1978), 75-83.

12. _ The Riemann Roch Theorem for complex V-manifolds, Osaka J. Math. 16 (1979), 151-159.

13. __ The index of elliptic operators over V-manifolds, Nagoya Math. J. 84 (1981), 135-157.

14. A. Miščenko and A. T. Fomenko, The index of elliptic operators over $C^{*}$-algebras, Math. USSR-Izv. 15 (1980), 87-112.

15. M. A. Rieffel, Applications of strong Morita equivalence to transformation group $C^{*}$ algebras, Operator Algebras and Applications (R. V. Kadison, ed.), Proc. Sympos. Pure Math., vol. 38, Amer. Math. Soc., Providence, RI, 1982, pp. 299-310.

16. I. Satake, The Gauss-Bonnet theorem for V-manifolds, J. Math. Soc. Japan 9 (1957), 464492.

Department of Mathematics, University of Colorado, Campus Box 426, Boulder, ColORADO, 80309

E-mail address: farsi@euclid.colorado.edu 